\title{
On the role of enthalpic and entropic contributions to the conforma- tional free energy landscape of MIL-101(Cr) secondary building units
}

\author{
Loukas Kollias $^{1}$, David C. Cantu ${ }^{2}$, Vassiliki-Alexandra Glezakou ${ }^{3 *}$, Roger Rousseau ${ }^{3}$, Matteo Salvalaglio ${ }^{1^{*}}$ \\ ${ }^{1}$ Thomas Young Centre and Department of Chemical Engineering, University College London, London WC1E 7JE, United Kingdom; \\ ${ }^{2}$ Chemical and Materials Engineering Department, University of Nevada, Reno, Reno, NV, 89557, United States; \\ ${ }^{3}$ Basic and Applied Molecular Foundations, Pacific Northwest National Laboratory, Richland, WA, 99352 United States \\ KEYWORDS: Molecular Dynamics, Metal-Organic Frameworks, Entropy
}

\begin{abstract}
The thermo-structural behavior of metal-organic framework (MOF) precursors is responsible for regulating the introduction of defects in MOF structures during synthesis. In this paper, factors affecting the flexibility of MIL-101(Cr) half-secondary building units (half-SBUs) are evaluated in solution using enhanced sampling methods. In particular, entropic and enthalpic contributions to the conformational free energy landscape of isolated MIL-101(Cr) half-SBUs are calculated in water, in the presence and absence of ionic species $\left(\mathrm{Na}^{+}\right.$and $\left.\mathrm{F}^{-}\right)$, and in $\mathrm{N}, \mathrm{N}$-dimethylformamide (DMF). This analysis leads to the observation that the interplay between enthalpy and entropy determines the most probable conformational state for half-SBUs. This observation extends to the most relevant SBU intermediate, in which conformational entropy plays a key role in stabilizing configurations that differ from those found in the MIL-101(Cr) crystal structure. Our findings highlight the importance of explicitly considering entropic effects, associated with finite-temperature sampling when estimating the relative stability of different conformers of SBUs.
\end{abstract}

\section{INTRODUCTION}

Questions around the enthalpic and entropic nature of interactions in solution are ubiquitous in the scientific literature. Notable examples of processes in which entropic contributions play a decisive role include peptide-metal nanoparticle interactions ${ }^{1-}$ ${ }^{3}$, surface stability $^{4}$, solute aggregation ${ }^{5}$, supramolecular . encapsulation $^{6}$ and preferential adsorption ${ }^{7}$. Entropy-enthalpy compensation phenomena in solvated molecules are key to understand how specific functional properties emerge from certain molecular structures ${ }^{1,6,8-12}$.

In the field of MOF synthesis, there is a growing effort to improve the mechanical stability of MOFs by taking advantage of novel synthetic techniques ${ }^{13-15}$. Mechanical flexibility and thermal expansion of MOFs have been investigated in previous works ${ }^{16-17}$. Computational and experimental studies on enthalpic and entropic contributions to the adsorption free energy of guest molecules on MOFs have been frequently carried out. ${ }^{18-21}$

Nevertheless, assessing the entropic and enthalpic contributions to the thermo-structural behavior of MOFs, is currently an underexplored area, which has the potential to uncover strategies for improving MOFs' intrinsic characteristics during synthesis. ${ }^{18}$ A key characteristic in this context is the density of defects, which should be minimized in MOF synthesis. ${ }^{22-25}$

In this work, we focus on estimating the role of entropic and enthalpic contributions to the configurational landscape of flexible precursors of MIL-101(Cr), namely secondary building units (SBUs) and half-SBUs. These building blocks play a primary role in directing MOF synthesis. For instance, Férey et al. identified a direct structural relation between soluble precursors (half-SBUs) and SBUs found in the crystal lattice, and connected the enthalpic and entropic contributions to precursors formation with structural rearrangements in the MOF lattice. ${ }^{26}$ Haouas et al. ${ }^{27}$ observed that half-SBUs and SBUs are the only necessary units to build a crystal. Also, Haouas compared MIL$101(\mathrm{Cr})$ synthesis in water against DMF and observed that solvent effects are key to determine the final MOF structure. ${ }^{22,28}$ Taddei et al. ${ }^{22}$ also described SBU formation as a key step in MOF synthesis. Working on MIL-53 Embrechts et al. ${ }^{29}$ identified the rearrangement of soluble MOF precursors as the ratelimiting step in MOF synthesis. The limited understanding of the behavior of molecular precursors of MOFs is a factor currently limiting the development of synthesis and material design strategies. ${ }^{30-32}$ For instance, Potter et al. recently suggested that the nature of conformational selectivity of SBUs is not yet fully understood and theoretical investigations should be carried out in this context. ${ }^{33}$

We recently proposed that the early stages of MOF nucleation are decisively influenced by the assembly of half-SBUs to produce SBUs. ${ }^{34-35}$ In particular, the rich conformational ensemble unveiled during the association of SBUs, highlights the importance of entropic contributions in defining the free energy landscape of MIL-101(Cr) precursors. ${ }^{34}$

Moreover, the propensity of healing defects in the crystal lattice, as well as the formation of crystal-like SBUs is impacted by solution composition. The relative abundance of different isomers of half-SBUs precursors, as well as the presence of spectator ions emerge as key variables in this context. ${ }^{34}$

In this work, we evaluate entropic-enthalpic contributions to the free energy profiles associated with structural fluctuations in half-SBUs and SBUs. In this context, configurational entropy is associated with molecular flexibility. ${ }^{36}$ Elucidating the contribution of enthalpy and entropy to the flexibility of MIL-101(Cr) precursors is key to understand assembly and thus material synthesis.

It should be noted that nucleation follows the formation of halfSBUs. ${ }^{35}$ These intermediates have been discussed in detail in 
previous works. ${ }^{34-35}$ In brief, half-SBUs MLA and MLB are stereo isomers and MLC is a structural isomer of both MLA and MLB. ${ }^{34}$ The SBU resulting from two MLA half-SBUs (AA SBU) dominates the equilibrium distribution of all possible SBUs combinations. ${ }^{34}$ Furthermore, the AA SBU is the largest source of crystal-like growth units building the MIL-101(Cr) lattice. ${ }^{34}$

This analysis allows for an evaluation of the conformational freedom that different intermediates experience in solution. This, in turn, provides a detailed description of their accessible structural fluctuations, and provides further insight into the nature of the ensemble of configurations sampled by precursors during self-assembly and nucleation.

\section{METHODS}

\section{Simulation setup}

Molecular dynamics (MD) simulations were performed in explicit solvent. The leapfrog integrator was used to propagate dynamics of the system with a time step of 2 fs. The $\operatorname{LINCS}^{37}$ algorithm preserved the distances of bonds involving hydrogen atoms. The cutoff for non-bonded interactions is $10 \AA$. Long range electrostatics were treated using the Particle-Mesh Ewald $(\mathrm{PME})^{38}$ scheme. The velocity rescaling thermostat developed by Bussi et al. ${ }^{39}$ and the Berendsen barostat ${ }^{40}$ preserved the temperature and pressure at $298 \mathrm{~K}$ and 1 bar respectively. To investigate higher temperature conditions, additional NVT simulations were performed at $493 \mathrm{~K}$, using the thermostat of Bussi et al. $^{39}$, consistent with MIL-101(Cr) experimental synthesis ${ }^{41}$. The system is simulated under a high pressure as in an autoclave employed during synthesis ${ }^{41}$. In more detail, the system of isolated half-SBUs (MLA, MLB and MLC) ${ }^{34-35}$ in solution, which consists of 6,500 atoms, was simulated in a cubic box of edge $40 \AA$ with periodic boundary conditions in three dimensions. The system of an SBU in solution has 12,000 atoms in a cubic cell with edge equal to $50 \AA$ A. Solvent has been treated explicitly with the TIP3P model ${ }^{42}$ and ions with the OPLS-AA force field $^{43}$. The force field for the half-SBU species is discussed in detail in a previous work ${ }^{34}$. Simulations were carried out using GROMACS $2018^{44}$ and PLUMED 2.5 ${ }^{45}$. Chemical structures were visualized using $\mathrm{VMD}^{46}$. All the data and PLUMED input files required to reproduce the results reported in this paper are available on PLUMED-NEST (www.plumed-nest.org), the public repository of the PLUMED consortium ${ }^{47}$, as plumID:19.073.

Well-tempered metadynamics (WTmetaD) ${ }^{48}$ is employed to enhance sampling and construct free energy surfaces along chemically intuitive collective coordinates. In this context, we construct a bias potential as a function of the mass-weighted radius of gyration $\left(\mathrm{R}_{\mathrm{gyr}}\right)$ to assess the flexibility of the MIL-101 $(\mathrm{Cr})$ half-SBUs ${ }^{35}$. The radius of gyration has been chosen as a low dimensional descriptor of the conformational state of halfSBUs. In particular we report the value of $\mathrm{R}_{\mathrm{gyr}}$ for each unit in the crystal on all our plots.

Gaussians of width $0.1 \AA$ and initial height $0.5975 \mathrm{kcal} \mathrm{mol}^{-1}$ were deposited every 1 ps with a bias factor of 10 in absence of ions, and a bias factor of 50 in presence of ions. The total simulation time in water in absence and presence of ions $(0.25 \mathrm{M})$, and DMF was $0.5 \mu$ s.
Furthermore, a simulation studying the rearrangement of an AA SBU in solution was performed through biasing two variables. The first variable is the number of terminal carboxylic oxygens with a coordination number with chromium atoms larger than 0.5 , using a coordination sphere of radius $2.5 \AA$. The second variable is the total potential energy of the system. Simulations were carried out for $1.5 \mu$ s at $298 \mathrm{~K}$ and $1.0 \mu$ s at $493 \mathrm{~K}$. Gaussians of width 0.01 and $47.8 \mathrm{kcal} \mathrm{mol}^{-1}$ for coordination number and potential energy were deposited every 1 ps with initial height and bias factor equal to $0.598 \mathrm{kcal} \mathrm{mol}^{-1}$ and 100 respectively. A rational switching function ${ }^{45}$ with parameters $\left(\mathrm{d}_{0}=0\right.$, $\mathrm{n}=2, \mathrm{~m}=4$ ) was used in order to calculate the set of coordination numbers. At last, a harmonic restraining potential $\left(V_{r}\right)$ acted on the lowest component of the set of all distances between any chromium and any terminal carboxylic carbon atoms. The potential, characterized by a force constant of $35.85 \mathrm{kcal} \mathrm{mol}^{-1}$ was active when the minimum distance exceeded $3.5 \AA$. Finally, we have calculated errors on the energy profiles due to sampling following a block analysis. A detailed description is available in the SI, Section VIII.

\section{Enthalpic and entropic contributions to the conformational free energy landscape.}

As a result of WTmetaD calculations, a free energy surface is the direct output, as a projection of the free energy of the system $G$ on the space of collective variables, denoted with $s .{ }^{48}$ The Gibbs free energy can be expressed as the sum of its enthalpic and entropic contributions as shown in Equation 1.

$\Delta \mathrm{G}(\boldsymbol{s})=\Delta H(\boldsymbol{s})-T \cdot \Delta S(\boldsymbol{s})$

where $G(s)$ is the free energy, $H(s)$ the enthalpy, and $S(s)$ the entropy, and $\Delta$ represents the difference with respect to an arbitrarily chosen reference state in $\mathbf{S}^{49}$.

To systematically decompose the free energy surface $\Delta G(\mathbf{s})$ we employed the method discussed in the work of Gimondi et al. ${ }^{49}$, which is based on mapping the ensemble average of the enthalpic component of the free energy in s, and then obtaining the entropic contribution to free energy by difference.

In the case of conformational transitions in solution, associated to negligible variations in the excluded volume, the $P \Delta V(s)$ contribution to enthalpy (where $P$ is the pressure and $V(s)$ the volume mapped on $\mathrm{CV}$ space) is constant. Hence $\Delta H(s)$ reduces to the internal energy $\Delta U(s)$. Furthermore, at constant $T$, the kinetic energy does not depend on $s$, and therefore the internal energy contribution $\Delta U(s)$ further reduces to the potential energy of the system $\Delta E_{\mathrm{P}}(s)^{49}$.

Since in this work we are analyzing conformational transitions in half-SBUs or SBUs embedded in a solvent, we shall highlight that the potential energy of the system is dominated by the potential energy of the solvent. To limit the statistical uncertainty in the decomposition of the free energy surface $\Delta G(s)$, we shall therefore further decompose the contributions to $\Delta E_{\mathrm{P}}(\mathbf{s})$ as follows in Equation 2.

$\Delta E_{P}(\mathbf{s})=\Delta\left\langle E_{P}^{\text {solute }}\right\rangle_{s}+\Delta\left\langle E_{P}^{\text {solute-solvent }}\right\rangle_{s}+\Delta\left\langle E_{P}^{\text {solvent }}\right\rangle_{s}+\Delta\left\langle E_{P}^{L R}\right\rangle_{s}(2)$

Where adopting the notation introduced in the work of Gimondi et al. ${ }^{49}\left\langle E_{P}^{\text {solute }}\right\rangle_{s}$, and $\left\langle E_{P}^{\text {solvent }}\right\rangle_{s}$ represent ensemble averages of the potential energy projected on $\boldsymbol{S}$, including all the bonded 
terms (bond, angles, proper dihedrals and Ryckaert-Bellemans dihedrals ${ }^{50}$ ) of solute and solvent respectively; plus the nonbonded, short-range terms for solute-solute and solvent-solvent interactions. Long-range interactions, $\left\langle E_{P}^{L R}\right\rangle$, are also accounted for all species.

The term $\left\langle E_{\mathrm{P}} \text { solute-solvent }\right\rangle_{s}$ accounts instead for the non-bonded, short-range potential energy terms associated to solute-solvent interactions. We shall note that the term $\left\langle E_{\mathrm{P}}{ }^{\text {solvent }}\right\rangle_{s}$ is independent from the conformation of the solute mapped on $s$, hence $\Delta\left\langle E_{\mathrm{P}}{ }^{\text {solvent }}\right\rangle_{s}=0$.

In order to compute $\Delta\left\langle E_{\mathrm{P}}{ }^{\text {solute }}\right\rangle_{s}$ and $\Delta\left\langle E_{\mathrm{P}}{ }^{\text {solute-solvent }}\right\rangle_{s}$ from biased sampling, we estimate Boltzmann weights using the method of Tiwary and Parrinello ${ }^{51}$.

\section{Solvation shell}

In order to investigate the role of the solvation shell on the enthalpy and entropy of rearrangement of half-SBUs, we calculate contributions to the potential energy by changing our convention for the molecules considered in the calculation of the term $\Delta\left\langle E_{P}^{\text {solute-solvent }}\right\rangle_{s}$.

In particular we now explicitly distinguish between solvent molecules found within a certain distance from the solute, constituting the solvation shell interacting with the half-SBU. By

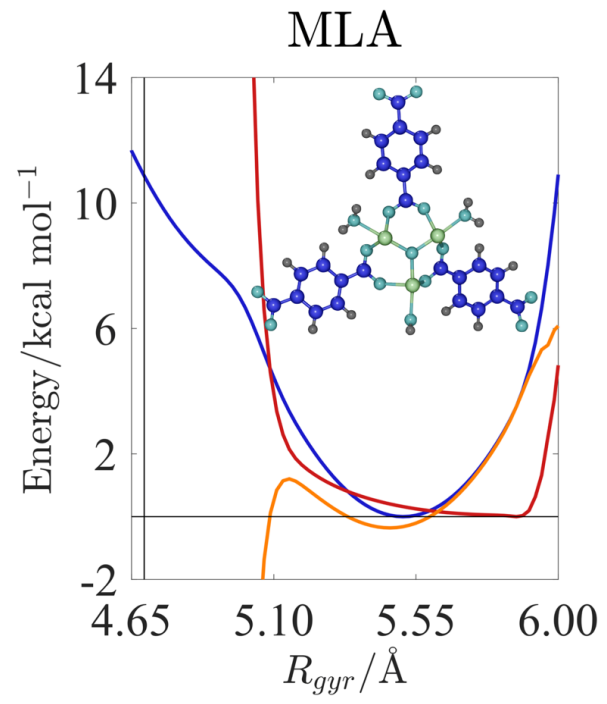

considering solvation shells of increasing size (from 2.5 to 20 $\AA$ ) and monitoring the convergence of the potential energy contribution $\Delta\left\langle E_{P}^{\text {solute-solvent }}\right\rangle_{s}$ as a function of the solvation shell size, we quantify the impact of the conformational rearrangement of half-SBUs on the surrounding solvent environment and assess solvation shell impact on the conformational entropy.

\section{Effect of ions and solvent}

The role of ions and the changes in the free energy and the enthalpy-entropy profiles are investigated. Ions $\left(\mathrm{Na}^{+}, \mathrm{F}^{-}\right)$are added $(0.25 \mathrm{M}$ with respect to the volume of the simulation box $)$, in order to evaluate half-SBU flexibility in excess of ionic species. We complement the interactions between the solute and the solvent with those between the ionic species and the solute. Furthermore, a different solvent can significantly affect the energetics of conformational transformations in the solute. This is due to entropic and enthalpic contributions being affected by the nature of solvation ${ }^{6}$. To assess this effect on MIL-101(Cr) SBUs, in addition to water we investigate N, N-dimethylformamide (DMF). The OPLS forcefield parameters for DMF were obtained from the virtualchemistry.org database $\mathrm{e}^{52-53}$.

Figure 1. Energy landscape of MLA, MLB and MLC in water at $298 \mathrm{~K}$ as a function of $\mathrm{R}_{\mathrm{gyr}}$. In blue we report the free energy profile, red: enthalpic contribution, orange: entropic contribution profile. Molecular configurations shown correspond to structures representative of the free energy minima in the respective profiles. Color code: Cr-lime, C-blue, O-cyan and H-gray. A thin horizontal line represents zero on the vertical axis. A black vertical line marks the average $\mathrm{R}_{\text {gyr }}$ value of a half-SBU in the bulk of a MIL-101(Cr) crystal.

\section{RESULTS}

We investigate the driving forces to the conformational complexity of the half-SBUs of MIL-101(Cr $)^{41}$ by exploring the energy profile as a function of $\mathrm{R}_{\mathrm{gyr}}$. We carry out this analysis for all three half-SBU isomers identified as key in the early stages of MIL-101(Cr) self-assembly ${ }^{35}$, computing the entropic and enthalpic contributions to free energy.

A first observation is that MLA has considerably different free energy and entropy minima, unlike MLB and MLC. In MLA, entropy favors compact configurations $\left(\mathrm{R}_{\mathrm{gyr}}=4.77 \AA\right)$, while enthalpy drives the system to larger values of $R_{\text {gyr }}(5.87 \AA)$. Higher temperature conditions $(493 \mathrm{~K})$ result to a similar free energy minimum compared to $298 \mathrm{~K}$. Enthalpy has a minimum at $5.73 \AA$ that is slightly less than at $298 \mathrm{~K}$. Entropy is dominated by considerably more compact, but less favorable in entropy space, structures than the minimum at $298 \mathrm{~K}\left(\mathrm{R}_{\mathrm{gyr}}=4.19\right.$ $\AA$ ). The profiles at $493 \mathrm{~K}$ are available in the SI, section V. MLB presents similar values of $R_{\text {gyr }}$ for the strongest enthalpic $\left(R_{\mathrm{gyr}}=5.73 \AA\right)$ and entropic contributions $\left(\mathrm{R}_{\mathrm{gyr}}=5.65 \AA\right)$ to the free energy, corresponding to the largest value of $R_{\text {gyr }}$ among the three half-SBUs, at $298 \mathrm{~K}$. MLC presents the minimum enthalpic contribution at a low value of $\mathrm{R}_{\mathrm{gyr}}(5.23 \AA$ ) ; hence rendering more compact configurations enthalpically favored. The 
entropic contribution has a minimum at $5.37 \AA$ at $298 \mathrm{~K}$. Another straightforward observation is that the minima for the enthalpic and entropic contributions correspond to similar values for $\mathrm{R}_{\mathrm{gyr}}$ as in MLB, while for MLA the respective $\mathrm{R}_{\mathrm{gyr}}$ values differ by more than $1 \AA$.

To summarize the analysis of the energy landscape for halfSBUs in water, the free energy of MLA is dominated by strongly contrasting enthalpic and entropic effects. Enthalpy favors "open" structures with stretched arms, while entropy favors more compact configurations. In MLB and MLC, enthalpy and entropy contribute in a similar manner to the free energy profile, with their minima exhibiting close values of $\mathrm{R}_{\mathrm{gyr}}$. We note that the average values (the standard deviation is provided in brackets) of $\mathrm{R}_{\mathrm{gyr}}$ in the crystal are 4.69 (0.16) $\AA$ for MLA, 5.44 (0.17) $\AA$ for MLB and 5.27 (0.13) $\AA$ for MLC. Therefore, entropy is the driver to the structures in the crystal for MLA and MLB. More elaborately, $\mathrm{R}_{\mathrm{gyr}}$ values corresponding to the minimum in the entropic contribution are closer to the average value in the crystal than those dominating the enthalpic contribution. In contrast, enthalpy is dominated by values of $R_{\mathrm{gyr}}$ closer to the ones in the crystal, than values corresponding to the minimum entropic contribution, only for MLC. The resulting energy profiles are reported in Figure 1.

\section{Effect of solvation shell}

The effect of the solvation shell on the entropic-enthalpic contribution profiles is investigated. Our motivation stems from the need of assessing the interplay between entropy and enthalpy in relation with solvation. For instance, strong enthalpic interactions between the solute and the solvent molecules, within a solvation shell, can induce significant changes in the entropy of these molecules against those far away from the solute ${ }^{9}$. Recently, Schauperl et al. ${ }^{54}$ showed that the water model choice can only result in minor differences in the entropy and enthalpy of solvation. Nevertheless, we have validated that our findings are not affected by this choice through calculating energy profiles using the TIP4P model ${ }^{42}$ as well (see SI, section VII).

We consider six solvation shell sizes: $2.5,5,7.5,10,15$ and 20 $\AA$. We note that entropy/enthalpy breakdown qualitatively converges for shell sizes larger than $5 \AA$. Calculating the differences in the enthalpy contribution at different shell sizes we conclude that a solvation shell of $7.5 \AA$ is sufficient to capture solute-solvent interactions. For instance, accounting for a solvation shell of size $7.5 \AA$ produces a maximum difference with respect to the enthalpy profile obtained including all the solvent molecules of the order of $k_{B} T$. Such a difference becomes negligible for solvation shell sizes larger than $10 \AA$, indicating that solute reorganization extends to solvent molecules within this distance. We note that the free energy profile is inherently associated with the configurational space of the entire simulation box, and it is not affected by changes in the size of the solvation shell.

An analogous analysis has been carried out for MLA in aqueous solution, in the presence of ions. Very similar trends are observed as in pure water; hence the solute-solvent interactions are fully captured within a solvation shell of size 7.5-10 $\AA$.

The enthalpy and entropy profiles computed as a function of solvation shell size are reported in the Supporting Information (SI), section IV.
In order to understand the effect of solvation on the enthalpic and entropic contributions to the free energy profiles, the radial distribution functions are calculated between the central oxygen of the metal center of each half-SBU $\left(\mathrm{O}_{\mathrm{u}}\right)$ and a characteristic atom of each solvent (the oxygen for water and the nitrogen for DMF). Radial distribution functions are reported in SI, section II. In water, the first solvation shell is located $3 \AA$ away from the central oxygen of MLA, in absence and presence of ions, and it contains 3 solvent molecules. The second solvation shell displays a bimodal structure, with the first peak located at 5.2 $\AA$. At $5.7 \AA$, water displays a second peak in the second solvation shell, while in the presence of ions the second solvation shell is represented by a monomodal peak centered at $5.7 \AA$. The third solvation shell in water is located between $7.5-8 \AA$ in water, both in presence and absence of counterions. This analysis highlights that the impact on the energetics of the system, induced by the conformational rearrangement of MLA extend up to the fourth coordination shell, involving up to 46 water molecules. Small discrepancies between the radial distribution functions in pure water and in water with ions are expected due to the presence of $\mathrm{F}^{-}$that perturbs the hydrogen bond network of water ${ }^{55}$.

In DMF, the first solvation shell is centered around $5.2 \AA$ with the second solvation shell located at $8 \AA$. This indicates that in DMF the solute-solvent interplay in the definition of the enthalpic and entropic contributions to the conformational free energy landscape only extends up to the second solvation shell, involving approximately 11 molecules.

\section{Addition of ions}

The addition of ions $(0.25 \mathrm{M})$ can significantly alter the energetics of the system due to their strong interactions with partially charged atoms of the half-SBU. Here we analyze contributions to the MLA free energy profile as this is the unit that contributes more significantly to the assembly of crystal-like SBUs. We observe that fluoride anions tend to interact strongly with the metal center. This leads to reduced interactions within the halfSBU and impacts fluctuations in the $\mathrm{R}_{\mathrm{gyr}}$. In particular we note that the free energy projected on the $\mathrm{R}_{\mathrm{gyr}}$ is considerably shallower with ions than in pure water (see Figure 2). Hence, we conclude that fluctuations in the apparent size of MLA in solution are facilitated by ions.

The use of a higher temperature (493 K, see Figure 2) results to a very similar, but narrower, free energy profile. In contrast, the enthalpic and entropic contributions present appreciable changes. Enthalpy has a minimum that corresponds to more open structures associated with larger $\mathrm{R}_{\mathrm{gyr}}$ values $(5.35 \AA$ at 298 $\mathrm{K}$ and $5.59 \AA$ at $493 \mathrm{~K}$ ). Entropy, instead, favors even more compact structures than at $298 \mathrm{~K}$ and it has a minimum at lower $\mathrm{R}_{\text {gyr }}$ values ( $4.65 \AA$ at $298 \mathrm{~K}$ vs. $4.49 \AA$ at $493 \mathrm{~K}$ ). Therefore, the increase of temperature results in highly contrasting enthalpic and entropic contributions to the free energy. An analogous analysis, and associated discussion for MLB and MLC halfSBUs is reported in the SI, section III. 


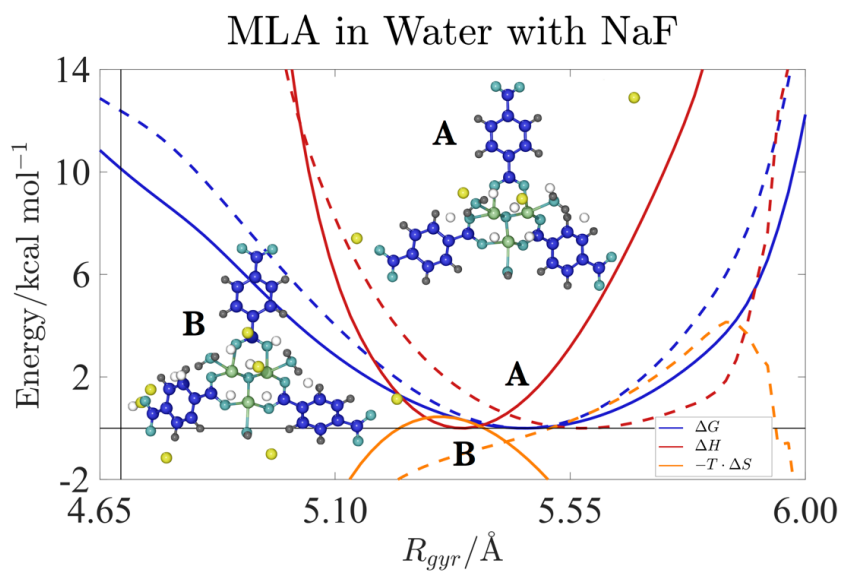

Figure 2. Energy landscape of MLA in water at $298 \mathrm{~K}$ (solid lines) and $493 \mathrm{~K}$ (dashed lines) with $\mathrm{NaF}(0.25 \mathrm{M})$ as a function of $\mathrm{R}_{\mathrm{gyr}}$. Blue: free energy, red: enthalpic contribution, orange: entropic contribution profile. Structure A corresponds to the free energy minimum and B to the enthalpic minimum at $298 \mathrm{~K}$. The color code is consistent with Figure 1. A black vertical line marks the average $\mathrm{R}_{\mathrm{gyr}}$ value of a half-SBU in the bulk of a MIL-101(Cr) crystal.

\section{Effect of solvent}

The effect of DMF becomes apparent for MLA when the potential energy is projected on collective variable space. In DMF, the free energy minimum is very close to the one obtained in water, but the underlying entropic and enthalpic contributions are considerably different. Both enthalpy and entropy profiles present a single basin in proximity to the free energy minimum and provide similar contributions to the overall free energy profile, as it can be seen in Figure 3.

We note that structural fluctuations away from the free energy minimum in the case of DMF do not require a high enthalpic cost. In other words, the enthalpy profile projected on $R_{\mathrm{gyr}}$ is shallow if compared with its analogous in water (see Figure 1, left panel). The entropic contribution to such fluctuations is even shallower, providing only a small contribution to the overall free energy, without significantly affecting the qualitative behavior of the system.

This is in stark contrast with the case of water, where fluctuations away from the free energy minimum are associated to a high enthalpic cost and are driven by the entropic contribution. Also, in this case the free energy profiles in DMF of MLB and MLC half-SBUs are analyzed in the SI section III.

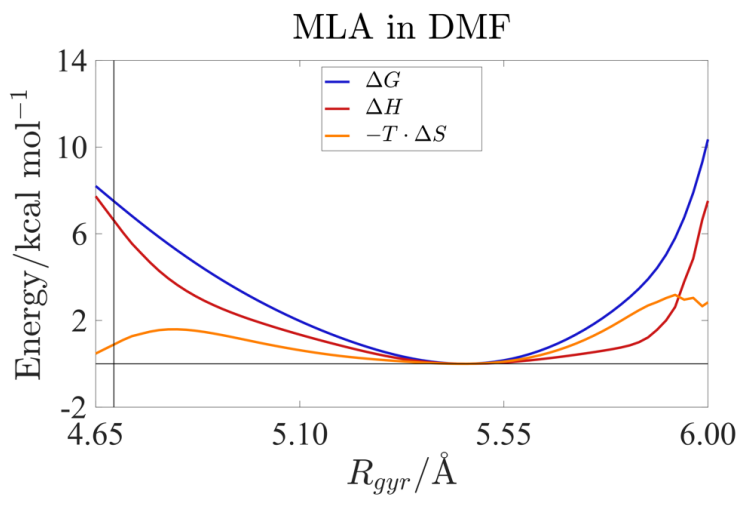

Figure 3. Energy landscape of MLA in DMF at $298 \mathrm{~K}$ as a function of $\mathrm{R}_{\mathrm{gyr}}$. Blue: free energy, red: enthalpic contribution, orange: entropic contribution profile. A thin horizontal line represents zero on the vertical axis. A black vertical line marks the average $\mathrm{R}_{\mathrm{gyr}}$ value of a half-SBU in the bulk of a MIL-101(Cr) crystal.

\section{Isomer symmetry}

Another important aspect that provides insight into the conformational flexibility of different isomers is a measure of symmetry along principal axes of inertia. The moments of inertia corresponding to rotational movement around principal axes are calculated for each half-SBU. We calculate the major, middle and minor axes of inertia and the corresponding moments. We observe that the major axis is similar for the three isomers. The middle and minor axes are appreciably different for MLA compared with the other two.

In water, the moment of inertia corresponding to the major axis has the highest most probable value for MLA and the lowest for MLC. Also, MLA and MLB populate a narrower range of values than MLC. The moment of inertia corresponding to the middle axis has the lowest most probable value for MLA and the highest for MLC. Again, MLA and MLB present a narrower range when compared with MLC. Finally, the moment of inertia corresponding to the minor axis presents a narrow distribution and the lowest most probable value for MLC. For MLB, the opposite occurs as it has the highest most probable value and a wide distribution. In addition to water, we assess the probability density of moments of inertia in DMF. Moments that correspond to the major and middle axes show very similar densities when compared with water, while rotation around the minor axis renders the total moment of inertia for MLA lower than in water and closer to MLC. This indicates that the molecule adopts a more compact structure that induces a smaller rotational inertia. This analysis offers an additional insight into the difference in the configurational ensemble populated by MLA in different solvents. Plots and additional details of this analysis are reported in the SI, section I.

\section{Study of the rearrangement of an SBU}

At last, we concentrate on the analysis of the entropic and enthalpic contributions to the free energy landscape associated to the conformational rearrangements of an SBU in solution.

For this analysis we focus on an SBU formed by two MLA halfSBUs. This SBU unit (indicated with the AA label) has emerged as the most important for the development of crystalline MIL$101(\mathrm{Cr}){ }^{34}$ 
We start the simulation from a crystal-like SBU and sample its configuration space biasing with WTmetaD the number of contacts between terminal carboxylic oxygens and chromium atoms. We repeat our calculation at two temperatures, 298 and $493 \mathrm{~K}$. A value of this collective variable equal to 1.6 corresponds to 2 chromium-oxygen interactions as found in the bulk of MIL-101(Cr). This configuration is labelled C for crystallike. A value of 2.5 , instead, is representative of 3 chromiumoxygen interactions, labelled $\mathrm{T}$ (for triple chromium-oxygen interaction). At last, a value of this collective variable equal to 3.3 , corresponds to 4 chromium-oxygen interactions and is labelled Q (indicating a quadruple chromium-oxygen interaction, corresponding to two chromium-oxygen interactions on each side).
We note that, at both temperature conditions, the free energy landscape underpinning the configurational rearrangement of the AA SBU, seen in Figure 4, is heavily affected by entropy. For instance, while $\mathrm{C}$ emerges as the minimum potential energy conformation, and is favored by enthalpy, Q emerges as an entropically dominated global free energy minimum. Finally, we note that, as expected, the entropically dominated Q configurations is further even more favored at higher temperatures. Free energy profiles including enthalpic and entropic contributions at both temperatures and structures characteristic of each state are available in Figure 4.

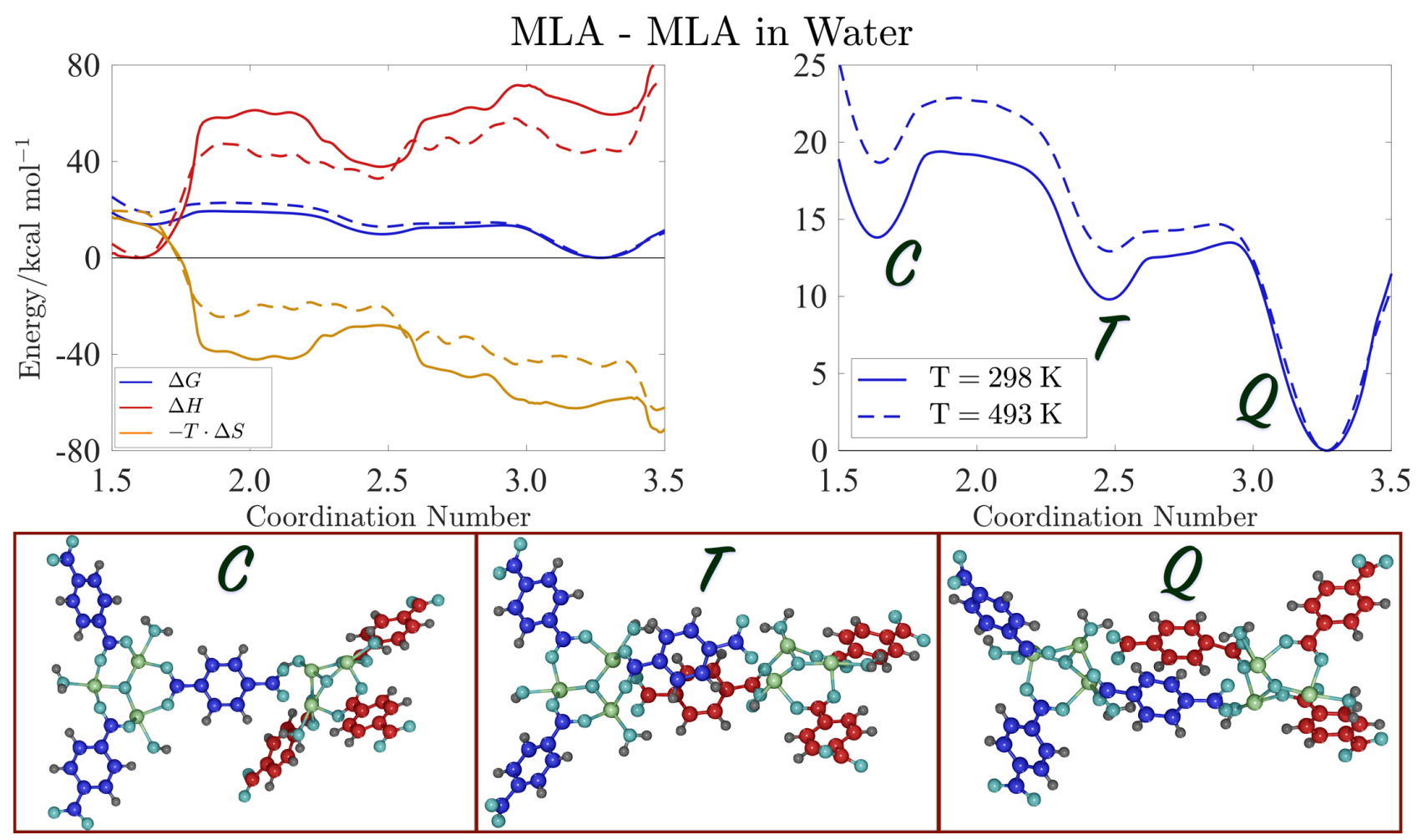

Figure 4. Energy landscape of the rearrangement of an AA SBU in water at $298 \mathrm{~K}$ (solid lines) and $493 \mathrm{~K}$ (dashed lines) with respect to the coordination number between terminal carboxylic oxygen and adjacent chromium atoms. Left panel: Blue: free energy, red: enthalpic contribution, orange: entropic contribution profile. Right panel: free energy profile in more detail. Configurations corresponding to the C, $\mathrm{T}$ and Q states are shown. The color code is consistent with Figure 1, except from carbon atoms that are colored blue for one half-SBU and red for its counterpart. A thin horizontal line represents zero on the vertical axis.

\section{CONCLUSIONS}

In this work we examine how different synthesis conditions affect the enthalpic and entropic contributions to the free energy landscape associated with structural fluctuations of MIL101(Cr) half-SBU isomers. In particular we focus on the impact of ions and solvent (water and DMF). The mass-weighted radius of gyration $\left(\mathrm{R}_{\mathrm{gyr}}\right)$ is used as a measure of isomer-specific molecular flexibility.

For MLA, the most important building block of MIL-101(Cr), we note that the presence of ions leads to larger structural fluctuations, captured by a shallower free energy profile function of
$\mathrm{R}_{\mathrm{gyr}}$, heavily affected by entropy. In contrast, the entropic contribution to free energy does not qualitatively impact the behavior of MLA in DMF, which compared to pure water also displays larger fluctuations that are driven by enthalpy.

Lastly, we analyze the conformational rearrangement of an SBU formed by two MLA units in water, which has been identified as key for determining the crystallinity of MIL-101(Cr) ${ }^{34}$. Our analysis shows that the crystal-like configuration of the SBU (C) is enthalpically favored, while SBU configurations displaying multiple concurrent linker-metal center interactions 
(T, Q) are entropically favored. Therefore, since entropy dominates the free energy landscape, a simple increase of temperature does not favor the development of crystalline domains. In fact, temperature renders the Q state even more probable as its free energy difference from configuration $\mathrm{C}$ is even larger. We thus identify configurational entropy as the main driver for the formation of SBUs that depart from a crystal-like configuration and are likely to result in high-defect materials. In a previous study, we showed how additive species, such as ions, drastically reduce the probability of observing configurations like $\mathrm{T}$ and $\mathrm{Q}^{34}$. Our current analysis suggests that in the case of full SBUs ions confine the accessible configuration space of building units by altering the entropy landscape. A synthetic strategy that carefully tunes the solution composition, would therefore allow the system to depart from an entropically dominated state, and increase the probability of forming crystal-like units. This observation provides a rationale for the use of ions during MOF synthesis ${ }^{41}$. From a computational standpoint, our findings highlight how static potential energy calculations provide only a partial and perhaps insufficient description of the configurational ensemble of MOF building units. Ultimately, this work provides new insights into synthetic strategies and optimizing synthesis conditions for entropically dominated processes.

\section{ASSOCIATED CONTENT}

\section{Supporting Information}

The Supporting Information is available free of charge on the ACS Publications website.

Supplementary information (SI) linked with the main article (PDF)

\section{AUTHOR INFORMATION}

\section{Corresponding Author \\ *vanda.glezakou@pnnl.gov \\ *m.salvalaglio@ucl.ac.uk}

\section{Notes}

The authors declare no competing financial interest.

\section{ACKNOWLEDGMENT}

The work described in this publication was performed by $\mathrm{Pa}$ cific Northwest National Laboratory (PNNL), which is operated by Battelle for the United States Department of Energy (DOE) under Contract DE-AC05-76RL0180. V.-A. G. and R. R. acknowledge support from the U.S. Department of Energy, Office of Science, Basic Energy Sciences, Chemical Sciences, Geosciences, and Biosciences Division. L. K. was partially supported by a Laboratory Directed Research and Development grant for PNNL. D.C.C. acknowledges support from Research and Innovation at the University of Nevada, Reno. The authors acknowledge the use of the UCL Myriad High Throughput Computing Facility (Myriad@UCL), and associated support services, in the completion of this work. We are grateful to the UK Materials and Molecular Modelling Hub for computational resources, which is partially funded by EPSRC (EP/P020194/1). This research used resources provided by the National Energy Research Scientific Computing Center (NERSC), a U.S. Department of Energy Office of Science User Facility operated under Contract No. DE-AC02-05CH11231.

\section{REFERENCES}

1. Choudhury, N.; Pettitt, B. M., Enthalpy-entropy contributions to the potential of mean force of nanoscopic hydrophobic solutes. J. Phys. Chem. B 2006, 110 (16), 8459-63.

2. van der Vegt, N. F. A.; van Gunsteren, W. F., Entropic Contributions in Cosolvent Binding to Hydrophobic Solutes in Water. J. Phys. Chem. B 2004, 108 (3), 1056-1064.

3. Tang, Z.; Palafox-Hernandez, J. P.; Law, W.-C.; Hughes, Z. E.; Swihart, M. T.; Prasad, P. N.; Knecht, M. R.; Walsh, T. R., Biomolecular Recognition Principles for Bionanocombinatorics: An Integrated Approach To Elucidate Enthalpic and Entropic Factors. ACS Nano 2013, 7 (11), 9632-9646.

4. Amirjalayer, S.; Tafipolsky, M.; Schmid, R., Surface Termination of the Metal-Organic Framework HKUST-1: A Theoretical Investigation. J. Phys. Chem. Lett. 2014, 5 (18), 32063210 .

5. Mondal, J.; Yethiraj, A., Driving Force for the Association of Amphiphilic Molecules. J. Phys. Chem. Lett. 2011, 2 (19), 23912395 .

6. Leung, D. H.; Bergman, R. G.; Raymond, K. N., Enthalpy-Entropy Compensation Reveals Solvent Reorganization as a Driving Force for Supramolecular Encapsulation in Water. J. Am. Chem. Soc. 2008, 130 (9), 2798-2805.

7. Van de Voorde, B.; Boulhout, M.; Vermoortele, F.; Horcajada, P.; Cunha, D.; Lee, J. S.; Chang, J.-S.; Gibson, E.; Daturi, M.; Lavalley, J.-C.; Vimont, A.; Beurroies, I.; De Vos, D. E., N/SHeterocyclic Contaminant Removal from Fuels by the Mesoporous Metal-Organic Framework MIL-100: The Role of the Metal Ion. J. Am. Chem. Soc. 2013, 135 (26), 9849-9856.

8. Eftink, M. R.; Anusiem, A. C.; Biltonen, R. L., Enthalpyentropy compensation and heat capacity changes for protein-ligand interactions: general thermodynamic models and data for the binding of nucleotides to ribonuclease A. Biochemistry 2002, 22 (16), 38843896.

9. Lumry, R.; Rajender, S., Enthalpy-entropy compensation phenomena in water solutions of proteins and small molecules: a ubiquitous property of water. Biopolymers 1970, 9 (10), 1125-227.

10. Mills, E. A.; Plotkin, S. S., Protein Transfer Free Energy Obeys Entropy-Enthalpy Compensation. J. Phys. Chem. B 2015, 119 (44), 14130-14144.

11. Pan, A.; Biswas, T.; Rakshit, A. K.; Moulik, S. P., EnthalpyEntropy Compensation (EEC) Effect: A Revisit. J. Phys. Chem. B 2015, 119 (52), 15876-15884.

12. Pan, A.; Kar, T.; Rakshit, A. K.; Moulik, S. P., EnthalpyEntropy Compensation (EEC) Effect: Decisive Role of Free Energy. $J$. Phys. Chem. B 2016, 120 (40), 10531-10539.

13. Moosavi, S. M.; Boyd, P. G.; Sarkisov, L.; Smit, B., Improving the Mechanical Stability of Metal-Organic Frameworks Using Chemical Caryatids. ACS Cent. Sci. 2018, 4 (7), 832-839.

14. Lal, G.; Derakhshandeh, M.; Akhtar, F.; Spasyuk, D. M.; Lin, J.-B.; Trifkovic, M.; Shimizu, G. K. H., Mechanical Properties of a Metal-Organic Framework formed by Covalent Cross-Linking of Metal-Organic Polyhedra. J. Am. Chem. Soc. 2018, 141 (2), $1045-$ 1053 .

15. Xie, X.-Y.; Wu, F.; Liu, X.; Tao, W.-Q.; Jiang, Y.; Liu, X.Q.; Sun, L.-B., Photopolymerization of metal-organic polyhedra: an efficient approach to improve the hydrostability, dispersity, and processability. Chem. Commun. 2019, 55, 6177-6180

16. Sarkisov, L.; Martin, R. L.; Haranczyk, M.; Smit, B., On the Flexibility of Metal-Organic Frameworks. J. Am. Chem. Soc. 2014, 136 (6), 2228-2231.

17. Balestra, S. R. G.; Bueno-Perez, R.; Hamad, S.; Dubbeldam, D.; Ruiz-Salvador, A. R.; Calero, S., Controlling Thermal Expansion: A Metal-Organic Frameworks Route. Chem. Mater. 2016, 28 (22), 8296-8304.

18. Torres-Knoop, A.; Dubbeldam, D., Exploiting Large-Pore Metal-Organic Frameworks for Separations through Entropic Molecular Mechanisms. ChemPhysChem 2015, 16 (10), 2046-2067. 
19. Torres-Knoop, A.; Poursaeidesfahani, A.; Vlugt, T. J. H.; Dubbeldam, D., Behavior of the Enthalpy of Adsorption in Nanoporous Materials Close to Saturation Conditions. J. Chem. Theory Comput. 2017, 13 (7), 3326-3339.

20. Gomez, D. A.; Sastre, G., From microscopic insights of H2 adsorption to uptake estimations in MOFs. Phys. Chem. Chem. Phys. 2011, 13 (37), 16558.

21. Couck, S.; Rémy, T.; Baron, G. V.; Gascon, J.; Kapteijn, F.; Denayer, J. F. M., A pulse chromatographic study of the adsorption properties of the amino-MIL-53 (Al) metal-organic framework. Phys. Chem. Chem. Phys. 2010, 12 (32), 9413.

22. Taddei, M., When defects turn into virtues: The curious case of zirconium-based metal-organic frameworks. Coord. Chem. Rev. 2017, 343, 1-24.

23. Howarth, A. J.; Liu, Y.; Li, P.; Li, Z.; Wang, T. C.; Hupp, J. T.; Farha, O. K., Chemical, thermal and mechanical stabilities of metal-organic frameworks. Nat. Rev. Mater. 2016, 1 (3), 15018.

24. Gutov, O. V.; Gonzalez Hevia, M.; Escudero-Adan, E. C.; Shafir, A., Metal-Organic Framework (MOF) Defects under Control: Insights into the Missing Linker Sites and Their Implication in the Reactivity of Zirconium-Based Frameworks. Inorg. Chem. 2015, 54 (17), 8396-400.

25. Howarth, A. J.; Peters, A. W.; Vermeulen, N. A.; Wang, T. C.; Hupp, J. T.; Farha, O. K., Best Practices for the Synthesis, Activation, and Characterization of Metal-Organic Frameworks. Chem. Mater. 2016, 29 (1), 26-39.

26. Férey, G.; Haouas, M.; Loiseau, T.; Taulelle, F., Nanoporous Solids: How Do They Form? An In Situ Approach. Chem. Mater. 2014, 26 (1), 299-309.

27. Haouas, M.; Volkringer, C.; Loiseau, T.; Férey, G.; Taulelle, F., In Situ NMR, Ex Situ XRD and SEM Study of the Hydrothermal Crystallization of Nanoporous Aluminum Trimesates MIL-96, MIL100, and MIL-110. Chem. Mater. 2012, 24 (13), 2462-2471.

28. Haouas, M., Nuclear Magnetic Resonance Spectroscopy for In Situ Monitoring of Porous Materials Formation under Hydrothermal Conditions. Materials 2018, 11 (8).

29. Embrechts, H.; Kriesten, M.; Hoffmann, K.; Peukert, W.; Hartmann, M.; Distaso, M., Elucidation of the Formation Mechanism of Metal-Organic Frameworks via in-Situ Raman and FTIR Spectroscopy under Solvothermal Conditions. J. Phys. Chem. C 2018 , 122 (23), 12267-12278.

30. Wagia, R.; Strashnov, I.; Anderson, M. W.; Attfield, M. P., Determination of the Preassembled Nucleating Units That Are Critical for the Crystal Growth of the Metal-Organic Framework CdIF-4. Angew. Chem. Int. Ed. 2016, 128 (31), 9221-9225.

31. Van Vleet, M. J.; Weng, T.; Li, X.; Schmidt, J. R., In Situ, Time-Resolved, and Mechanistic Studies of Metal-Organic Framework Nucleation and Growth. Chem. Rev. 2018, 118 (7), 36813721 .

32. Xu, H.; Sommer, S.; Broge, N. L. N.; Gao, J.; Iversen, B. B., The Chemistry of Nucleation: In Situ Pair Distribution Function Analysis of Secondary Building Units During UiO-66 MOF Formation. Chem. Eur. J. 2019, 25 (8), 2051-2058.

33. Potter, M. E.; Light, M. E.; Irving, D. J. M.; Oakley, A. E.; Chapman, S.; Chater, P.; Cutts, G.; Watts, A.; Wharmby, M.; Vandegehuchte, B. D.; Schreiber, M. W.; Raja, R., Exploring the origins of crystallisation kinetics in hierarchical materials using in situ $\mathrm{X}$-ray diffraction and pair distribution function analysis. Phys. Chem. Chem. Phys. $\mathbf{2 0 2 0 .}$

34. Kollias, L.; Cantu, D. C.; Tubbs, M. A.; Rousseau, R.; Glezakou, V. A.; Salvalaglio, M., Molecular Level Understanding of the Free Energy Landscape in Early Stages of Metal-Organic Framework Nucleation. J. Am. Chem. Soc. 2019, 141 (14), 6073-6081.
35. Cantu, D. C.; McGrail, B. P.; Glezakou, V. A., Formation Mechanism of the Secondary Building Unit in a Chromium Terephthalate Metal-Organic Framework. Chem. Mater. 2014, 26 (22), 6401-6409.

36. Brady, J.; Karplus, M., Configuration entropy of the alanine dipeptide in vacuum and in solution: a molecular dynamics study. $J$. Am. Chem. Soc. 1985, 107 (21), 6103-6105.

37. Hess, B.; Bekker, H.; Berendsen, H. J. C.; Fraaije, J. G. E. M., LINCS: A linear constraint solver for molecular simulations. $J$. Comput. Chem. 1997, 18 (12), 1463-1472.

38. Darden, T.; York, D.; Pedersen, L., Particle Mesh Ewald an N.Log(N) Method for Ewald Sums in Large Systems. J. Chem. Phys. 1993, 98 (12), 10089-10092.

39. Bussi, G.; Donadio, D.; Parrinello, M., Canonical sampling through velocity rescaling. J. Chem. Phys. 2007, 126 (1), 014101.

40. Berendsen, H. J. C.; Postma, J. P. M.; Vangunsteren, W. F.; Dinola, A.; Haak, J. R., Molecular-Dynamics with Coupling to an External Bath. J. Chem. Phys. 1984, 81 (8), 3684-3690.

41. Férey, G.; Mellot-Draznieks, C.; Serre, C.; Millange, F.; Dutour, J.; Surble, S.; Margiolaki, I., A chromium terephthalate-based solid with unusually large pore volumes and surface area. Science $\mathbf{2 0 0 5}$, 309 (5743), 2040-2.

42. Jorgensen, W. L.; Chandrasekhar, J.; Madura, J. D.; Impey, R. W.; Klein, M. L., Comparison of Simple Potential Functions for Simulating Liquid Water. J. Chem. Phys. 1983, 79 (2), 926-935.

43. Jorgensen, W. L.; Tirado-Rives, J., The OPLS [optimized potentials for liquid simulations] potential functions for proteins, energy minimizations for crystals of cyclic peptides and crambin. $J$. Am. Chem. Soc. 1988, 110 (6), 1657-66.

44. Abraham, M. J.; Murtola, T.; Schulz, R.; Páll, S.; Smith, J. C.; Hess, B.; Lindahl, E., GROMACS: High performance molecular simulations through multi-level parallelism from laptops to supercomputers. SoftwareX 2015, 1-2, 19-25.

45. Tribello, G. A.; Bonomi, M.; Branduardi, D.; Camilloni, C.; Bussi, G., PLUMED 2: New feathers for an old bird. Comput. Phys. Commun. 2014, 185 (2), 604-613.

46. Humphrey, W.; Dalke, A.; Schulten, K., VMD - Visual Molecular Dynamics. J. Mol. Graph. 1996, 14, 33-38.

47. The PLUMED consortium, Promoting transparency and reproducibility in enhanced molecular simulations. Nat. Methods 2019, $16(8), 670-673$.

48. Barducci, A.; Bussi, G.; Parrinello, M., Well-tempered metadynamics: a smoothly converging and tunable free-energy method. Phys. Rev. Lett. 2008, 100 (2), 020603.

49. Gimondi, I.; Tribello, G. A.; Salvalaglio, M., Building maps in collective variable space. J. Chem. Phys. 2018, 149 (10), 104104.

50. Ryckaert, J.-P.; Bellemans, A., Molecular dynamics of liquid alkanes. Faraday Discuss. 1978, 66, 95.

51. Tiwary, P.; Parrinello, M., A time-independent free energy estimator for metadynamics. J. Phys. Chem. B 2015, 119 (3), 736-42.

52. Caleman, C.; van Maaren, P. J.; Hong, M.; Hub, J. S.; Costa, L. T.; van der Spoel, D., Force Field Benchmark of Organic Liquids: Density, Enthalpy of Vaporization, Heat Capacities, Surface Tension, Isothermal Compressibility, Volumetric Expansion Coefficient, and Dielectric Constant. J. Chem. Theory Comput. 2012, 8 (1), 61-74.

53. van der Spoel, D.; van Maaren, P. J.; Caleman, C., GROMACS molecule \& liquid database. Bioinformatics 2012, 28 (5), $752-753$.

54. Schauperl, M.; Podewitz, M.; Waldner, B. J.; Liedl, K. R., Enthalpic and Entropic Contributions to Hydrophobicity. J. Chem. Theory Comput. 2016, 12 (9), 4600-4610.

55. Gallo, P.; Corradini, D.; Rovere, M., Do ions affect the structure of water? The case of potassium halides. J. Mol. Liq. 2014, 189, 52-56. 
Graphic entry for the Table of Contents (TOC)

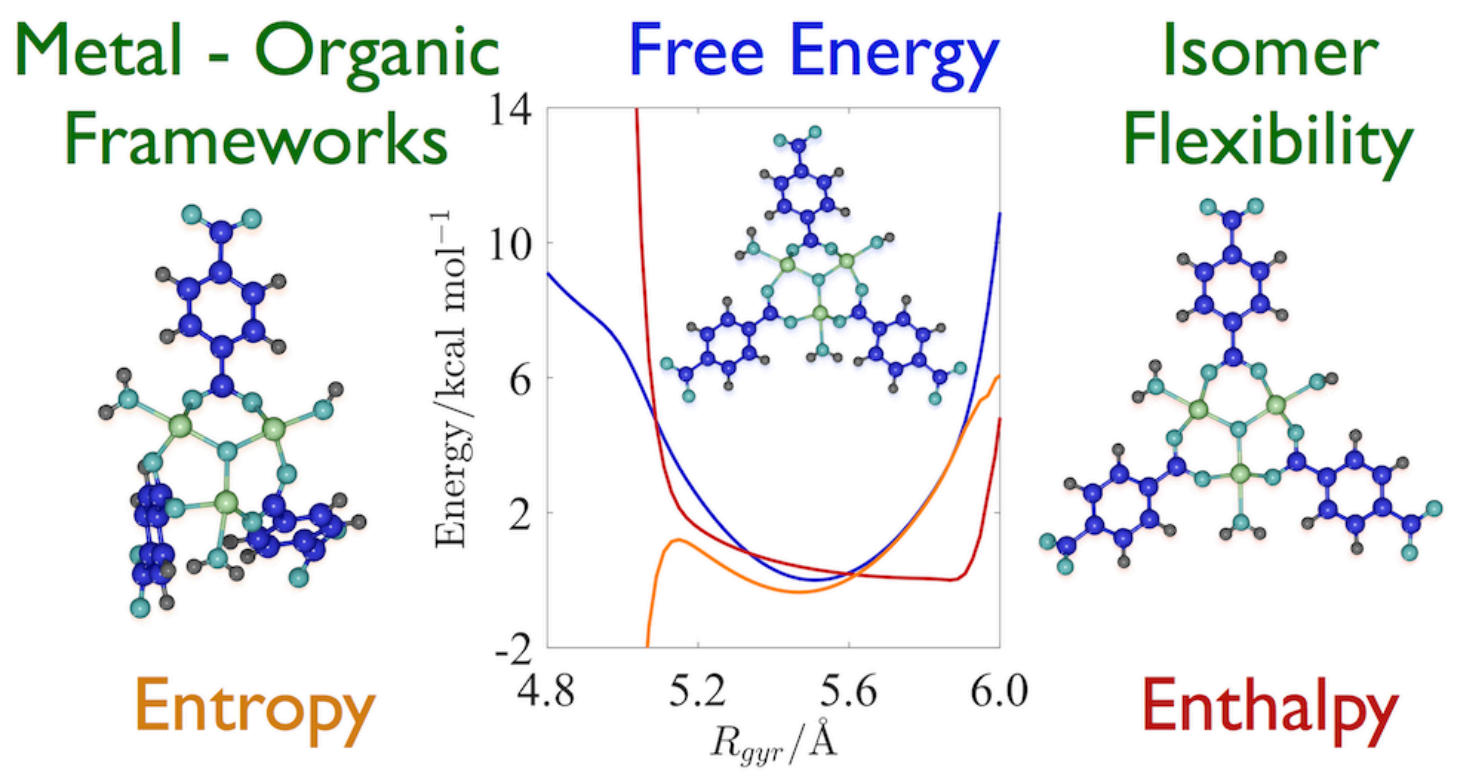

